Check for updates

Cite this: RSC Adv., 2018, 8, 41472

Received 16th October 2018

Accepted 3rd December 2018

DOI: $10.1039 / c 8 r a 08557 a$

rsc.li/rsc-advances

\section{Tunable two-dimensional polarization grating using a self-organized micropixelated liquid crystal structure $\uparrow$}

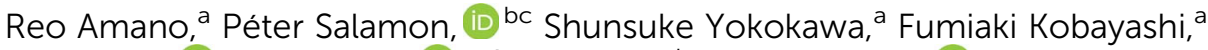 \\ Yuji Sasaki, (D) *a Shuji Fujii, (D) a Ágnes Buka, ${ }^{\text {b }}$ Fumito Araoka (D) \\ and Hiroshi Orihara (iD)
}

Utilization of the self-organizing nature of soft materials is promising for fabricating micro- and nanostructures, which can be applied for optics. Because of the high birefringence, liquid crystals are especially suitable for optoelectronic applications such as beam steering and polarization conversion. On the other hand, most self-organized patterns in liquid crystals are one-dimensional and there are only a few examples of two dimensional systems. Here we study the light diffraction from a micro-pixelated pattern of a nematic liquid crystal which is formed by self-organization of topological defects. We demonstrate that the system works as a tunable two dimensional optical grating, which splits the incident laser beam and changes the polarization property. The intensity can be controlled by electrical voltages, which cause extinction of the zeroth-order beam. The polarization properties depend on the location of spots. The numerical calculation and the theoretical analysis not only support the experimental results but also unveil the uniqueness of the pixelated structure.

\section{Introduction}

Nematic liquid crystals (NLCs) are anisotropic fluids in which elongated molecules are aligned in a preferred direction called the director, $\boldsymbol{n}$. Because the optical anisotropy can be controlled by electric fields, NLCs are suitable for optoelectronic applications such as displays. LC gratings are interesting examples, which are targeted to beam steering and optical filters. ${ }^{1-8}$ In particular, LC gratings can show high first-order diffraction efficiency and excellent polarization-separation properties. ${ }^{1,3,5,9-12}$ In order to fabricate gratings using LCs, a periodic modulation of director field in a small area is essentially required. Several methods have been proposed so far to realize high-resolution control of $\boldsymbol{n}$ for various LCs applications. Common approaches are to use top-down lithographic processes such as pre-patterned electrodes, ${ }^{1,11,13,14}$ scribing the substrate by AFM, ${ }^{15}$ and photo-alignment. ${ }^{9,16-18}$ Another candidate is the bottom-up approach that uses a self-organized periodic pattern., ${ }^{2,6,19-21}$ It is known that LCs generally exhibit

${ }^{a}$ Division of Applied Physics, Faculty of Engineering, Hokkaido University, North 13 West 8, Kita-ku, Sapporo, Hokkaido 060-8628, Japan. E-mail: yuji.sasaki@eng. hokudai.ac.jp; Tel: +81 (0)11 7066642

${ }^{b}$ Institute for Solid State Physics and Optics, Wigner Research Centre for Physics, Hungarian Academy of Sciences, H-1525 Budapest, P. O. Box 49, Hungary

'RIKEN Center for Emergent Matter Science (CEMS), 2-1 Hirosawa, Wako, Saitama 351-0198, Japan

$\dagger$ Electronic supplementary information (ESI) available. See DOI: 10.1039/c8ra08557a rich pattern formation ${ }^{22-24}$ under external stimuli such as the electro-hydrodynamic effect ${ }^{25,26}$ and by the helical structure of the chiral NLCs. ${ }^{27-29}$ However these patterns are mostly limited to one-dimensional striped structure. Two dimensional patterns are relatively rare $^{23-26}$ and moreover it is difficult to obtain a mono-domain structure in a large area. Thus, the diffraction properties of two dimensional self-organized patterns have not been investigated thoroughly. In this regard, recently, by doping an NLC with ionic additives, we have found a two-dimensional micropattern, which is self-organized by applying electric fields. ${ }^{30}$ The pattern consists of a square array of topological defects, which are often called umbilics. ${ }^{31,32}$ The umbilical defect structure is induced when homeotropically aligned NLC molecules with negative dielectric anisotropy are reoriented. Because this system does not require a prepatterned surface, it is possible to change both the size of the unit cell and the birefringence with external fields. Thus, such a self-organized system is interesting for optical applications. One particular exploitation is the generation of an optical vortex from the defect structure. ${ }^{33,34}$ We have recently reported optical vortex generation using this two-dimensional grid pattern. ${ }^{35}$ There, we have simultaneously observed light diffraction. However, in the earlier paper, the diffraction has not been understood in detail.

In this paper, we present the results of our comprehensive investigation aiming to understand light diffraction from the micro-pixelated LC pattern. We uncover how the diffraction pattern depends on the effective birefringence and on the 
frequency of the driving AC electrical voltage. The polarization state of the diffracted light is also studied. The experiments show that the intensity of diffraction spots can be controlled by the reorientation of the director field. Particularly, high diffraction-efficiency is realized under a moderate electrical voltage, which extincts the zeroth order spot. We also find that the polarization of diffracted light is converted differently depending on the diffraction order. In addition to experiments, we perform numerical calculations to understand the voltage dependence, which shows a qualitative agreement with the experimental results. The presented experimental and numerical results are understood theoretically, which also reveals the uniqueness of this pixelated structure.

\section{Experimental}

Fig. 1 shows the schematic illustration of a sample cell. Using two ITO (indium tin oxide)-coated glass plates, standard sandwich type cells are prepared. The ITO coating is stripe patterned with $1.2 \mathrm{~mm}$ wide and the substrates are spin-coated with a perfluoropolymer (CYTOP, Asahi Glass Co.). The thickness of the alignment layer is around $120 \mathrm{~nm}$. Two substrates are overlapped so that the ITO stripes cross orthogonally each other. The mono-dispersed micro-spheres are used to maintain a proper thickness of the sample. From our earlier work, it is known that the obtained grid size is proportional to the sample thickness. ${ }^{30}$ Here, we use the sample cells with the gap of $13 \mu \mathrm{m}$ and $21 \mu \mathrm{m}$. An NLC ( $4 \alpha, 4^{\prime} \alpha$-propylheptyl-1 $\alpha, 1^{\prime} \alpha$-bicyclohexyl- $4 \beta$ carbonitrile, CCN-37, Fig. 1(b)) doped with $1 \mathrm{wt} \%$ of an ionic additive (tetrabutylammonium benzoate, TBABE) is filled into the cell by capillary action. $\mathrm{CCN}-37$ possesses a negative dielectric anisotropy $(\Delta \varepsilon \sim-7)$ and the birefringence $(\Delta n)$ is $0.03 .{ }^{36}$ At room temperature, $\mathrm{CCN}-37$ shows the homeotropic alignment at the CYTOP surface. ${ }^{37}$ Then, by applying an ac electrical voltage $V=V_{0} \cos (2 \pi f t)$, the director reorientation leads to the formation of a square array of umbilical defects in our system. A monodomain can be obtained by adjusting the electrical voltage. We use a He-Ne laser (633 nm in wavelength) to investigate the diffraction property. The monodomain formed in the area of $1.2 \mathrm{~mm} \times 1.2 \mathrm{~mm}$ is large enough compared to the beam size of $0.7 \mathrm{~mm}$ in diameter. If necessary, wave plates and/or polarizers are placed in front of and behind the sample cell. The power of the diffracted light is measured with a power meter (Thorlabs PM100 USB) for each spot which is seen on the screen. The diffraction is captured with a CMOS
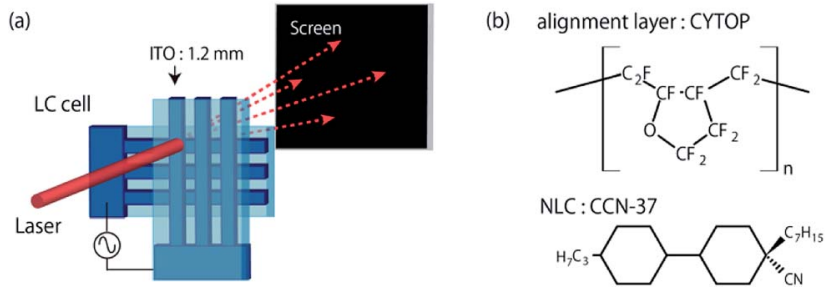

Fig. 1 (a) Schematic illustration of the sample cell and the diffraction experiments. (b) The chemical structures of the alignment layer (CYTOP) and the NLC (CCN-37). camera (Thorlabs DCC1240C). Before starting the diffraction measurements, both the texture of the pattern and the irradiation position of laser beam are checked with polarized optical microscopy (POM). The microscopy is installed on the optical path so that the POM observation is possible without disarranging the optical setup.

\section{Results and discussion}

\section{Experimental results}

A POM image of the pattern is displayed in Fig. 2(a). The cell thickness is $13 \mu \mathrm{m}$. To induce the director reorientation, we increase the frequency, $f$ under a constant amplitude, $V_{0}$. The range of $f$ is chosen not to break the pattern. The change in the micrographic appearance by the increase of $f$ is shown in Fig. 2(b) and (c). Due to the enhanced retardation, the brightness changes under the same illumination condition. After preparing the suitable monodomain, a laser beam is irradiated (a)

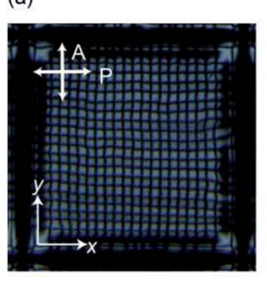

$11.5 \mathrm{~Hz}$

(d)

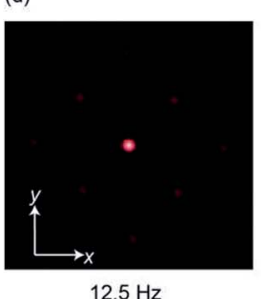

(g)

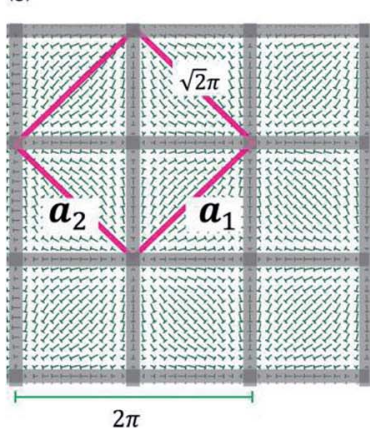

(b)

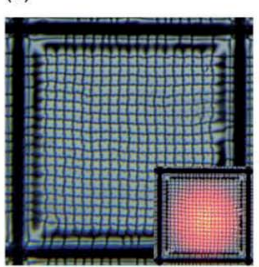

$15.5 \mathrm{~Hz}$

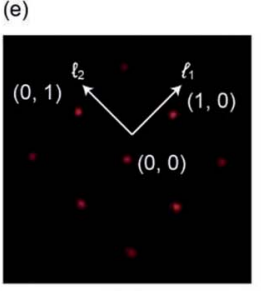

$18.5 \mathrm{~Hz}$ (c)

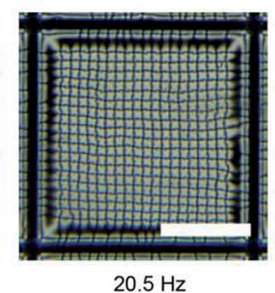

(f)

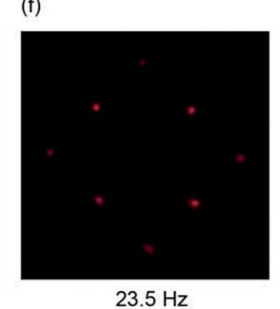

(h)

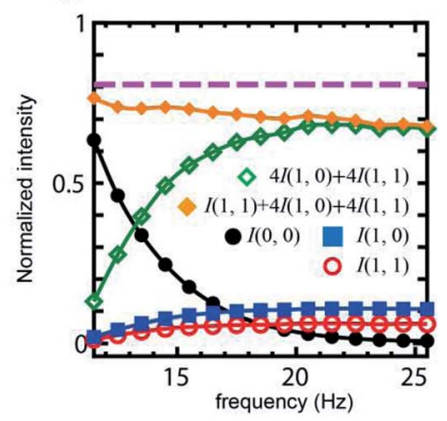

Fig. 2 (a)-(c) The micrographic images for the grid-like texture under cross-polarized condition. Scale bar, $500 \mu \mathrm{m}$. The inset of (b) is a micrograph of a grid-like texture irradiated with a laser beam. (d)-(f) Diffraction patterns obtained by a laser beam irradiation. The incident beam is circularly polarized. The amplitude of the applied voltage is $25 \mathrm{~V}$. (d) $12.5 \mathrm{~Hz}$, (e) $18.5 \mathrm{~Hz}$, (f) $23.5 \mathrm{~Hz}$. (g) A schematic illustration of the director field of the grid-like texture. The square is the unit cell for the light diffraction. Here, we set the lattice constant as $\sqrt{2} \pi$ without loss of generality. (h) The intensity for each spot as a function of frequency. The intensity is normalized with the incident beam. The dashed line is the total power of the light transmitted through the cell. The power meter is placed right behind the cell. 
to the sample cell. The inset of Fig. 2(b) is an image at which a linearly polarized laser beam is irradiated. The position of the laser beam on the pattern is checked. We see that the texture of the irradiated area is not damaged, indicating that the heating effect is negligible. The diffraction pattern obtained by a circularly polarized light is shown in Fig. 2(d)-(f). We can see a square array of spots whose direction is tilted by $45^{\circ}$ from the horizontal axis. This clearly indicates that the primitive cell of the diffraction pattern corresponds to the square region displayed in Fig. 2(g) as already suggested in our recent study. ${ }^{35} \mathrm{We}$ denote the primitive cell vectors by $\boldsymbol{a}_{1}, \boldsymbol{a}_{2}$. The primitive reciprocal vectors $\boldsymbol{b}_{1}$ and $\boldsymbol{b}_{2}$ are defined as $\boldsymbol{a}_{i} \cdot \boldsymbol{b}_{j}=2 \pi \delta_{i j}$ : $\boldsymbol{a}_{i}$ is parallel to $\boldsymbol{b}_{i}$ in our case. With $\boldsymbol{b}_{1}$ and $\boldsymbol{b}_{2}$, the spot $\left(\ell_{1}, \ell_{2}\right)$ is expressed as $\ell_{1} \boldsymbol{b}_{1}+\ell_{2} \boldsymbol{b}_{2}$ as shown in Fig. 2(e). Here we focus on nine spots $\{(0$, $0),( \pm 1,0),(0, \pm 1),( \pm 1, \pm 1),( \pm 1, \mp 1)\}$. We do not study higher order spots because their intensity is quite weak. With a constant $V_{0}$, the effect of the frequency for the diffraction pattern is shown in Fig. 2(d)-(f). It is seen that the intensity at each spot exhibits the frequency dependence. In the low frequency, i.e., in the beginning of the pattern formation from the homeotropic alignment, the zeroth-order spot $I(0,0)$ is dominant. This is obvious because the director field is almost perpendicular to the cell substrate. As the frequency increases, the intensity $I(0,0)$ decreases, while surrounding spots become reinforced. For $\sim 20 \mathrm{~Hz}$, the zeroth-order diffraction spot almost disappears (Fig. 2(f)), i.e., a high diffraction efficiency is realized. Further increase of $f$ does not change the brightness of the spots. This indicates that the director in the cell is well oriented and the retardation cannot be increased further in this cell. To obtain the quantitative data, the intensity of the spots is plotted as a function of frequency. (Fig. 2(h)) The intensity is normalized with that of the incident light $(40 \mu \mathrm{W})$. As seen from the diffraction pattern, $I(0,0)$ decreases. For higher frequency $(\geq 20$ $\mathrm{Hz}$ ), the change in the intensity is small. A crude estimation is made for how much light is split. We approximate the total power of the diffracted light as $I(0,0)+4(I(1,0)+I(1,1))$. The behavior of $4(I(1,0)+I(1,1))$ which is the surrounding bright spots, demonstrates that $I(0,0)$ is diffracted to the surrounding spots efficiently. Considering that the transmitted light behind the substrate amounts around $80 \%$, we find that $87 \%$ of the transmitted light is diffracted for the surrounding eight spots when $I(0,0)$ takes the minimum value and the rest amount is used for higher-order spots.

In addition to the effect of the electrical voltage for the light intensity, the polarization properties are studied by placing an analyzer behind the sample cell. Typical results are summarized in Fig. 3, which uses a cell with the thickness of $21 \mu \mathrm{m}$. POM observation under crossed-polarizers shows that the size of the grid becomes large compared to Fig. 2 because it is proportional to the sample thickness. ${ }^{30}$ By increasing the frequency, POM images show a significant color alternation due to the change of the retardation. (Fig. 3(b) and (c)) Considering the birefringence of $\Delta n \sim 0.03$, the possible maximum value of $\Delta n d$ is $\sim 600 \mathrm{~nm}$ if the director configuration is planar. This can be seen as a blue color under POM, which qualitatively agrees with the observation. This suggests that the director is substantially tilted in the sample cell around $25 \mathrm{~Hz}$. The incident light is circularly (a)

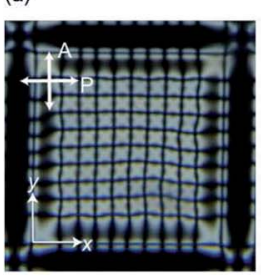

$11 \mathrm{~Hz}$
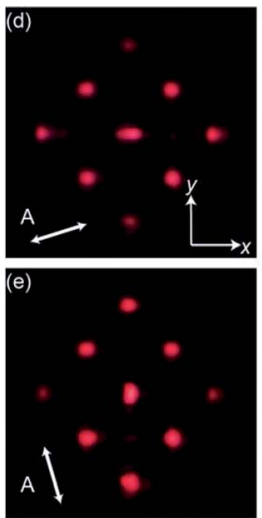

(g)

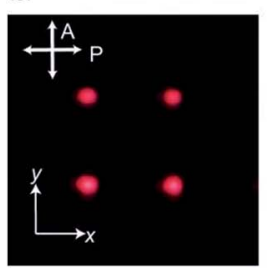

(b)

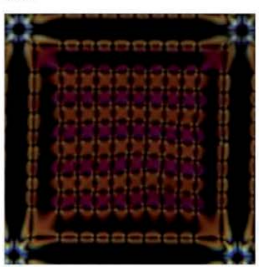

$18 \mathrm{~Hz}$ (c)

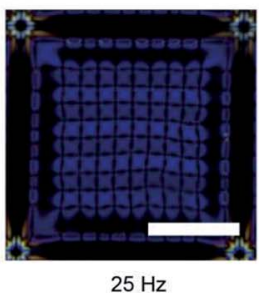

(f)

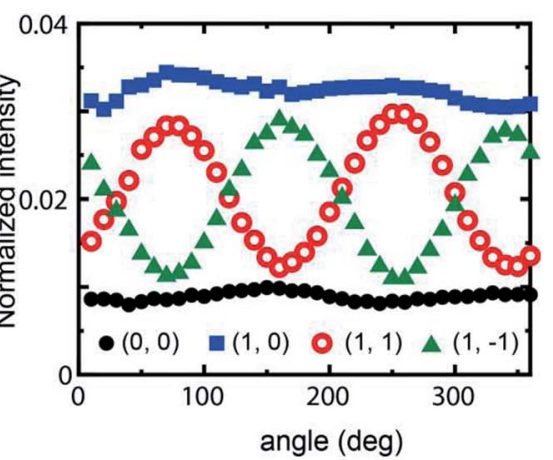

(h)

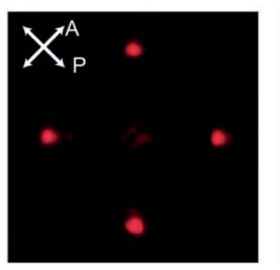

(i)

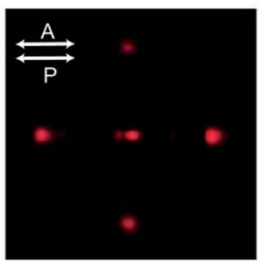

Fig. 3 (a)-(c) POM images for a cell with the sample thickness of 21 $\mu \mathrm{m} . V_{0}=25 \mathrm{~V} . f=11 \mathrm{~Hz}$ for (a), $18 \mathrm{~Hz}$ for (b), and $25 \mathrm{~Hz}$ for (c). Scale bar, $500 \mu \mathrm{m}$. (d) and (e) Diffraction patterns obtained by rotating the analyzer. The incident light is circularly polarized. (f) The angle dependence for the intensity of diffraction spots. Note that the experimental conditions in (f) and the other diffraction patterns ( $(d)$ and (e)) are different. (g)-(i) Diffraction patterns obtained by using a linearly polarized light.

polarized as used in Fig. 2. The diffraction pattern exhibits a similar trend to that of $13 \mu \mathrm{m}$ cell. When the director tilts from the homeotropic alignment, the value of $I(0,0)$ approaches zero because the light is split. (ESI Fig S1 $\dagger$ ) An additional feature observed for the $21 \mu \mathrm{m}$-thick cell is that the decreased $I(0,0)$ is reinforced from the minimum value by increasing the frequency. This is because the retardation can be varied over a wide range compared to the $13 \mu \mathrm{m}$-thick cell. Fig. 3(d) and (e) show that the brightness changes depending on the direction of analyzer. We notice that there are two types of spots whose intensity is independent $\{(0,0),( \pm 1,0),(0, \pm 1)\}$ and dependent $\{( \pm 1, \pm 1),( \pm 1, \mp 1)\}$ from the direction of analyzer. This means that the polarization of the diffracted light is converted differently depending on the spots. We also see that the quality of the diffracted spots is slightly low compared to the $13 \mu \mathrm{m}$-thick cell. For example, the zeroth order spot is distorted with the direction of the analyzer. However, this is due to the imperfection of the grid-like structure and the decreased number of the grids in the irradiated area. In order to investigate details, the frequency 
is adjusted at a moderate value so that all the spots exhibit comparable brightness. The intensity at each spot is plotted as a function of the direction of analyzer in Fig. 3(f). We note that the low value in the normalized intensity is due to the presence of the polarizers which absorb the light. For the spots of $\{(0,0)$, $( \pm 1,0),(0, \pm 1)\}$, we can expect that the diffracted light is circularly polarized. On the other hand, the spots of $\{( \pm 1, \pm 1)$, $( \pm 1, \mp 1)\}$ indicate that the polarization is elliptical. For these four spots, the angular dependence of the intensity of $( \pm 1, \pm 1)$ and $( \pm 1, \mp 1)$ is opposite, i.e., if $I( \pm 1, \pm 1)$ decrease, $I( \pm 1, \mp 1)$ increase. The phase shift for $\{( \pm 1, \pm 1),( \pm 1, \mp 1)\}$ varies by the applied electric field. Further experiments are carried out using a linearly polarized light. The diffraction pattern is studied under cross- and parallel-polarized conditions. (Fig. 3(g)-(i)) The zeroth-order spot can be erased as far as the cross-polarized condition is kept. For the other diffraction spots, the brightness changes by rotating crossed-polarizers. If a polarizer is set parallel to $x$-axis, four spots at $( \pm 1,0),(0, \pm 1)$ are obtained. On the other hand, when the crossed-polarizers are rotated by $45^{\circ}$, the spots $( \pm 1,0),(0, \pm 1)$ disappear and the spots of $( \pm 1, \pm 1)$, $( \pm 1, \mp 1)$ appear. Thus, $I(0,0)$ and other spots $( \pm 1,0),(0, \pm 1)$ which are independent of the direction of analyzer (Fig. 3(f)), have different property of polarization conversion. The different conversion can be found under parallel polarized condition. We find that $I( \pm 1,0)$ and $I(0, \pm 1)$ cannot be observed. These observations indicate that the conversion at $( \pm 1,0)$ and $(0, \pm 1)$ is the same as that of the half-wave plate whose optical axis is directed to the origin.

\section{Numerical calculation}

To understand the observation, we calculate the diffraction numerically by using the Jones matrix method. This approach is valid because the diffraction angle of this experiment is typically around 0.01 rad. Firstly, we estimate the effective retardation in two dimension and map it to the projected director field. It is to be noted that the maximum retardation which is the value at the center of each grid, increases together with the spatial change of the director field by increasing the frequency. Then, to describe the frequency dependence, we express the retardation as $\Delta n d=$ $\left(n_{\mathrm{e}}{ }^{\text {eff }}(\boldsymbol{r})-n_{0}\right) d=\delta_{0} F(\boldsymbol{r})=\delta(\boldsymbol{r})$ where $\boldsymbol{r}=(x, y) . \delta_{0}$ is the maximum value of the retardation at the center of each grid and $F(x, y)$ is a function which qualitatively reproduces the effect of the director tilts. Supposing that the cell thickness is $20 \mu \mathrm{m}, \delta_{0} \leq$ $600 \mathrm{~nm}$ and $0 \leq F(\boldsymbol{r}) \leq 1$ are imposed. Using Jones matrix $J(\boldsymbol{r})$ for the LC cell, the diffraction pattern at the screen is calculated with $\tilde{\boldsymbol{E}}(\boldsymbol{k}) \propto \int J(\boldsymbol{r}) \boldsymbol{E}(\boldsymbol{r}) \exp (-i \boldsymbol{k} \cdot \boldsymbol{r}) d \boldsymbol{r}$. We assume that the incoming laser beam $\boldsymbol{E}(\boldsymbol{r})$ is uniform over the irradiated area, i.e., $\boldsymbol{E}(\boldsymbol{r})=$ $\boldsymbol{E}_{\text {in }}$. Then $\tilde{\boldsymbol{E}}(\boldsymbol{k}) \propto \int J(\boldsymbol{r}) \exp (-i \boldsymbol{k} \cdot \boldsymbol{r}) d \boldsymbol{r} \cdot \boldsymbol{E}_{\text {in }}$. When two optical axes of the director field are parallel to $x$ - and $y$-axis, the Jones matrix $J^{\prime}$ is given as

$$
J^{\prime}=\left(\begin{array}{cc}
\exp \left(i \frac{2 \pi n_{\mathrm{e}}^{\mathrm{eff}}(\boldsymbol{r}) d}{\lambda}\right) & 0 \\
0 & \exp \left(i \frac{2 \pi n_{0} d}{\lambda}\right)
\end{array}\right) .
$$

$n_{\mathrm{e}}{ }^{\text {eff }}(\boldsymbol{r})$ varies depending on $\boldsymbol{r}$. Then we obtain

$$
J^{\prime}=\exp \left(i \frac{2 \pi n_{0} d}{\lambda}\right)\left(\begin{array}{cc}
\exp (i \Gamma) & 0 \\
0 & 1
\end{array}\right)
$$

where $\Gamma=2 \pi \Delta n d / \lambda$. By neglecting the constant term, $J(\boldsymbol{r})$ is expressed as

$$
J(\boldsymbol{r})=R(\theta(\boldsymbol{r}))\left(\begin{array}{cc}
\exp (i \Gamma) & 0 \\
0 & 1
\end{array}\right) R(-\theta(\boldsymbol{r})) .
$$

Here $\theta(\boldsymbol{r})$ is the rotation angle of the director from $x$-axis. $R(\theta)$ is the rotation matrix, which is given as

$$
R(\theta)=\left(\begin{array}{cc}
\cos \theta & -\sin \theta \\
\sin \theta & \cos \theta
\end{array}\right)
$$

Using $\left(n_{x}, n_{y}\right)$, we obtain $\tan ^{-1}\left(n_{y} / n_{x}\right)$. To describe the director field shown in Fig. $2(\mathrm{~g})$, we use a vector field of

$$
\left(n_{x}, n_{y}\right) \propto(\sin x, \sin y) .
$$

First, we examine the case for the constant retardation of $F(x$, $y)=1$, i.e., $\Delta n d=\delta_{0}$. This basically corresponds to the planar alignment with defects. Some calculated diffraction patterns are plotted in Fig. 4(a) and (b), where circularly polarized input light is used. The appearance seems to reproduce the experiments. We change the value of the retardation, which qualitatively corresponds to the application of the electric field. (Fig. 4(c)) We find that the diffraction efficiency changes depending on the retardation of the sample. For small $\delta_{0}$, the zeroth-order spot $I(0,0)$ is dominant and the surrounding spots are weak. At $2 \delta_{0}=$ $\lambda, I(0,0)$ vanishes and the diffraction efficiency reaches $100 \%$. The surrounding spots increase the intensity. When $\delta_{0}$ is increased further, $I(0,0)$ increases again and the surrounding spots become darker. The intensity for $(1,0)$ is higher than that for $(1,1)$. The tendency agrees with the experiments. On the other hand, a contrast is found in the polarization properties (Fig. 4(d)). The intensity at each spot is obtained by placing analyser behind the sample. In contrast to the experiments, the calculation does not show the angular dependence of the rotating analyser for all the spots. This means that the split light is circularly polarized even for $(1, \pm 1)$ and $(-1, \pm 1)$ when the alignment is planar. The calculated diffraction pattern under crossed- and parallel-polarizers is shown in Fig. 4(e) and (f). The appearance is similar to that of the experiment. Thus, we notice that the spots of $( \pm 1,0)$ and $(0, \pm 1)$ behave as a half wave plate whose optical axis is directed to $x$ - and $y$-axes. Thus, as suggested from the experiments, when the polarization of the incident light is parallel to $x$-axis, it can pass through the analyzer even in a cross-polarized condition. From the above, we find that the planar alignment condition qualitatively explains the experiments, while the obtained numerical results are incomplete in part.

In our experimental system, the retardation varies depending on the reorientation of the director. To evaluate the influence of umbilical defects qualitatively, we use a function $F(x, y)$ $=\left(2-\cos ^{n} x-\cos ^{n} y\right) / 2$ for effective value. $n$ takes an even 
(a)

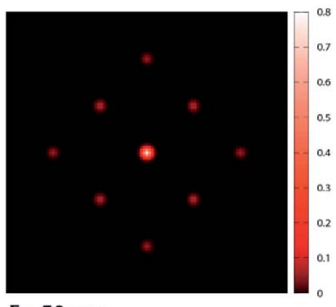

$\bar{\delta}_{0}=50 \mathrm{~nm}$

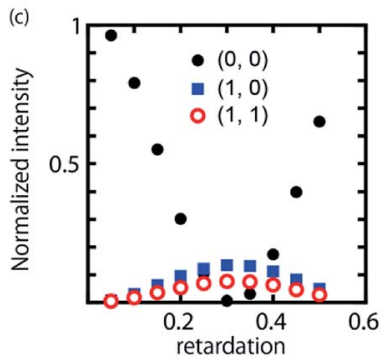

(e)

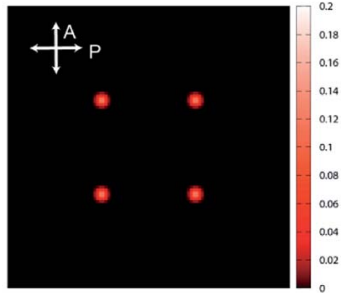

$\bar{\delta}_{0}=317 \mathrm{~nm}$

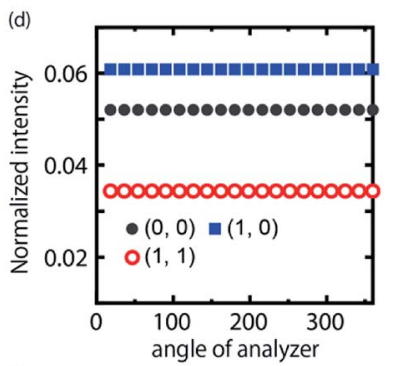

(f)

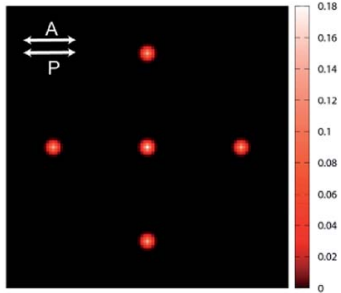

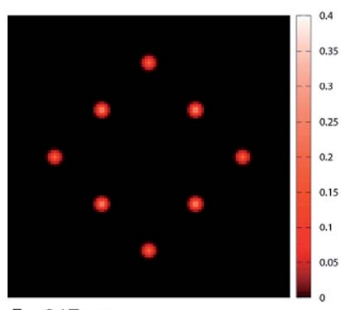

Fig. 4 Calculated diffraction patterns for the planar alignment condition using circularly polarized input light and retardation of (a) 50 and (b) $300 \mathrm{~nm}$. (c) The behavior of the intensity depending on the retardation. (d) The polarization dependence for the spot. Here the calculation is made for $\Delta n d=250 \mathrm{~nm}$. (e) and (f) Calculated diffraction patterns under crosspolarized (e) and parallel polarized (f) conditions.

number. For a small $n$, this corresponds to the beginning of the formation of the grid-like texture, while further tilted configuration can be described with a larger $n$. We consider the effects by changing $\delta_{0}$ and $n$. In Fig. 5(a), we show a calculation which is made with $\delta_{0}=350 \mathrm{~nm}$ and $n=40$. An important finding is that for $( \pm 1, \pm 1),( \pm 1, \mp 1)$, the polarization property is modified, which agrees with the experiments. This means that the elliptically polarized light at these spots is due to the spatial
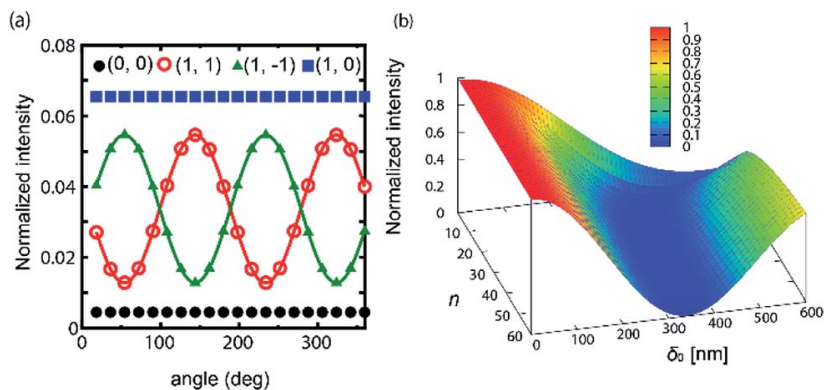

Fig. 5 (a) The angular dependence of the intensity of diffraction spots. The value of intensity is normalized with that of the incident light. The calculation is made with $\delta_{0}=350 \mathrm{~nm}$ and $n=40$. (b) The intensity of $I(0,0)$ as a function of $n$ and $\delta_{0}$. modulation of the birefringence. Moreover, from the simulated results, we can elucidate the director field of the sample. For the presented case, it is suggested that the director field changes suddenly near the core of the defect and the other region has a uniform tilt. We also check the behavior of $I(0,0)$, which is plotted as the function of $\delta_{0}$ and $n$. (Fig. 5(b)) When $n$ has a larger value, the graph shows a minimum. In particular, $I(0,0)$ becomes close to 0 for a high value of $n$. Similar to the case of the planar configuration (Fig. $4(\mathrm{c})$ ), we notice that $I(0,0)$ exhibits a minimum point around $\delta_{0} \sim \lambda / 2$. This demonstrates that our experimental system can also realize nearly $100 \%$ diffraction efficiency.

\section{Theoretical consideration}

We discuss the results of diffraction patterns from a symmetrical point of view. Taking into account that two director states $\left(n_{x}, n_{y}, n_{z}\right)$ and $\left(n_{x}, n_{y},-n_{z}\right)$ are optically the same, i.e., the corresponding Jones matrices are the same, the grid-like pattern belongs to the two-dimensional space group $P 4 m$. The symmetry elements are shown in Fig. 6. The symmetry operation can be expressed as $\boldsymbol{r}^{\prime}=\hat{T} \boldsymbol{r}=T \boldsymbol{r}+\boldsymbol{t}$, where $T$ is a matrix representing a rotation or a mirror (reflection) with respect to the origin, and $\boldsymbol{t}$ is a translation vector. A Jones matrix $J(\boldsymbol{r})$ is transformed to $T J(\boldsymbol{r}) T^{-1}$ by $\hat{T}$ as the Jones matrices are second rank tensors, and $\boldsymbol{r}$ is moved to $T \boldsymbol{r}+\boldsymbol{t}$. Since $\hat{T}$ is a symmetry operator, we have

$$
J(T \boldsymbol{r}+\boldsymbol{t})=T J(\boldsymbol{r}) T^{-1}
$$

The Jones matrix in the Fourier space is defined as

$$
\tilde{J}(\boldsymbol{k})=S_{\mathrm{p}}^{-1} \int_{S_{\mathrm{p}}} J(\boldsymbol{r}) \exp (-i \boldsymbol{k} \cdot \boldsymbol{r}) \mathrm{d} \boldsymbol{r}
$$

where the integration is taken over the primitive cell defined by two primitive vectors $\boldsymbol{a}_{1}$ and $\boldsymbol{a}_{2}$ shown in Fig. 6 and $S_{\mathrm{p}}$ is its area. Fourier transformation of eqn (3) yields

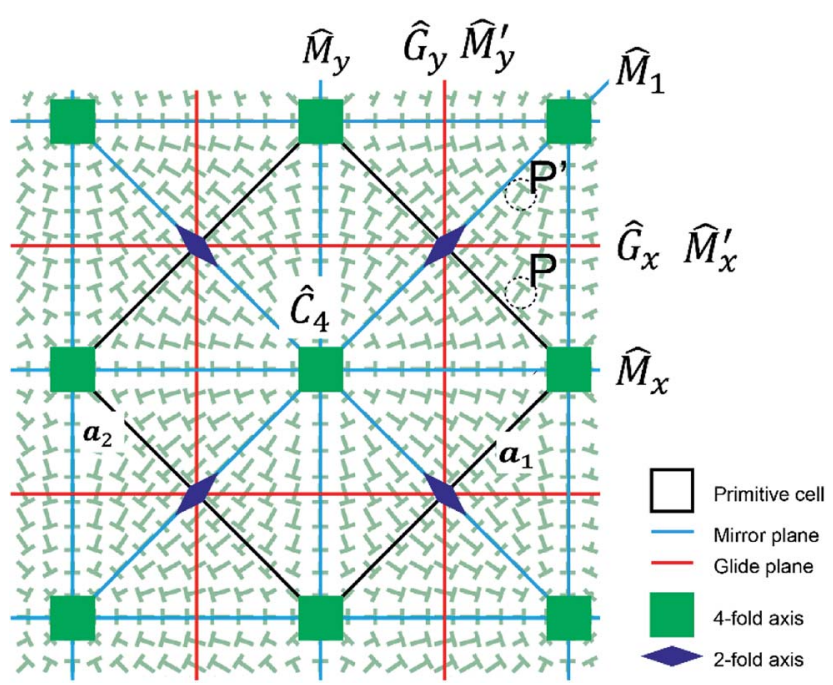

Fig. 6 Schematic illustration of the symmetry operation for this system. 


$$
\tilde{J}(T \boldsymbol{k}) \exp (-i T \boldsymbol{k} \cdot \boldsymbol{t})=T \tilde{J}(\boldsymbol{k}) T^{-1}
$$

In the following, we examine the symmetry of $\tilde{J}(\boldsymbol{k})$ at some $\boldsymbol{k}$ based on eqn. ${ }^{5}$ For simplicity, we use the director field of Fig. $2(\mathrm{~g})$ because the following results are valid independent of specific director fields as far as the symmetry is $P 4 m$. First, we consider a symmetry operation with a mirror $\hat{M}_{1}$ (i.e., $\hat{T} \boldsymbol{r}=\hat{M}_{1} \boldsymbol{r}$ $\left.=M_{1} \boldsymbol{r}+\boldsymbol{t}\right)$ which is the diagonal direction in Fig. 6. Here, $M_{1}$ and $\boldsymbol{t}$ are given as

$$
M_{1}=\left(\begin{array}{ll}
0 & 1 \\
1 & 0
\end{array}\right) \text { and } \boldsymbol{t}=0 .
$$

For $\boldsymbol{k}=\boldsymbol{b}_{1}$, we notice that $M_{1} \boldsymbol{b}_{1}=\boldsymbol{b}_{1}$. By using

$$
\tilde{J}\left(M_{1} \boldsymbol{b}_{1}\right)=\tilde{J}\left(\boldsymbol{b}_{1}\right)=\left(\begin{array}{ll}
e & f \\
g & h
\end{array}\right),
$$

eqn (5) becomes

$$
\left(\begin{array}{ll}
e & f \\
g & h
\end{array}\right)=\left(\begin{array}{ll}
0 & 1 \\
1 & 0
\end{array}\right)\left(\begin{array}{ll}
e & f \\
g & h
\end{array}\right)\left(\begin{array}{ll}
0 & 1 \\
1 & 0
\end{array}\right)
$$

which leads to

$$
\tilde{J}\left(\boldsymbol{b}_{1}\right)=\left(\begin{array}{cc}
e & f \\
f & e
\end{array}\right) .
$$

The symmetric form of Jones matrix indicates that for the diffracted light at $\boldsymbol{b}_{1}$ the sample plays a role of a wave-plate whose principal axes (the optical axes) are diagonal between $x$ - and $y$-axes. To obtain $\tilde{J}\left(-\boldsymbol{b}_{2}\right)$, we use another symmetry operation which rotates the director by $\frac{\pi}{2}$ with respect to $z$-axis. $\left(\hat{C}_{4}\right)$ Taking $\boldsymbol{k}=\boldsymbol{b}_{1}$, we obtain from eqn (5)

$$
\begin{aligned}
\tilde{J}\left(\boldsymbol{b}_{2}\right) & =C_{4} \tilde{J}\left(\boldsymbol{b}_{1}\right) C_{4}^{-1}=\left(\begin{array}{cc}
0 & -1 \\
1 & 0
\end{array}\right)\left(\begin{array}{cc}
e & f \\
f & e
\end{array}\right)\left(\begin{array}{cc}
0 & 1 \\
-1 & 0
\end{array}\right) \\
& =\left(\begin{array}{cc}
e & -f \\
f & e
\end{array}\right) .
\end{aligned}
$$

In the same way, the operation of $\hat{C}_{4}$ can be applied for $\tilde{J}\left(-\boldsymbol{b}_{1}\right)$ and $\tilde{J}\left(-\boldsymbol{b}_{2}\right)$, which leads to

$$
\begin{gathered}
\tilde{J}\left(-\boldsymbol{b}_{1}\right)=\tilde{J}\left(\boldsymbol{b}_{1}\right) \\
\tilde{J}\left(-\boldsymbol{b}_{2}\right)=\tilde{J}\left(\boldsymbol{b}_{2}\right)
\end{gathered}
$$

Second, we consider $\boldsymbol{k}=\boldsymbol{b}_{1}-\boldsymbol{b}_{2}$. Using a mirror operator in $x$-direction $\left(\tilde{M}_{x}\right)$, eqn (5) gives

$$
\tilde{J}\left(\boldsymbol{b}_{1}-\boldsymbol{b}_{2}\right)=\left(\begin{array}{cc}
e & 0 \\
0 & h
\end{array}\right)
$$

and further successive operation of $\hat{C}_{4}$ gives rise to the followings:

$$
\begin{array}{r}
\tilde{J}\left(\boldsymbol{b}_{1}+\boldsymbol{b}_{2}\right)=\left(\begin{array}{cc}
h & 0 \\
0 & e
\end{array}\right), \\
\tilde{J}\left(-\boldsymbol{b}_{1}+\boldsymbol{b}_{2}\right)=\tilde{J}\left(\boldsymbol{b}_{1}-\boldsymbol{b}_{2}\right),
\end{array}
$$

$$
\tilde{J}\left(-\boldsymbol{b}_{1}-\boldsymbol{b}_{2}\right)=\tilde{J}\left(\boldsymbol{b}_{1}+\boldsymbol{b}_{2}\right) .
$$

At these diffraction spots, the optical axes are in $x$ - and $y$ directions.

Last, we show only the result at $\boldsymbol{k}=0$,

$$
\left(\begin{array}{ll}
e & 0 \\
0 & e
\end{array}\right)
$$

which gives optically isotropic properties.

From experiments and numerical calculations, for $\boldsymbol{k}=\boldsymbol{b}_{1}$, the property of the polarization conversion is the same as that of the half-wave plate whose optical axes are directed to the origin. This indicates that $e$ of $\tilde{J}\left(\boldsymbol{b}_{1}\right)$ in eqn (6) should vanish. The abovementioned symmetry never leads to the expected result. However, we notice another symmetry (this is not usual symmetry) in Fig. 6, that is, the tilt angles at $P$ and $P^{\prime}$ are the same, which is mutually exchanged by the mirror $\hat{M}_{x}^{\prime}$ (note that $\hat{M}_{x}^{\prime}$ is different from the glide $\hat{G}_{x}$, although they are in the same line.). $\hat{M}_{y}^{\prime}$ is also this type of operator. In general, these operators do not exist although they do in our case of $\left(n_{x}, n_{y}\right) \propto(\sin x$, $\sin y)$. It is easily confirmed that they exist for $\left(n_{x}, n_{y}\right) \propto(g(x)$, $g(y))$ with $g(x)=\sum_{n=0}^{\infty} a_{n} \sin (2 n+1) x$, where $a_{n}$ are arbitrary constants. For $\hat{M}_{x}^{\prime}$ the following equation is valid instead of eqn (5) as $\tilde{J}$ does not change by $\hat{M}_{x}^{\prime}$

$$
\begin{gathered}
\tilde{J}\left(M_{x}^{\prime} \boldsymbol{k}\right) \exp \left(i M_{x}^{\prime} \boldsymbol{k} \cdot \boldsymbol{t}\right)=\tilde{J}(\boldsymbol{k}) \\
\tilde{M}_{x}^{\prime}=\left(\begin{array}{cc}
1 & 0 \\
0 & -1
\end{array}\right) \\
\boldsymbol{t}=\frac{1}{2}\left(\boldsymbol{a}_{1}+\boldsymbol{a}_{2}\right)
\end{gathered}
$$

Substitution of $\boldsymbol{k}=\boldsymbol{b}_{1}$ into eqn (14) yields $-\tilde{J}\left(-\boldsymbol{b}_{2}\right)=\tilde{J}\left(\boldsymbol{b}_{1}\right)$. Using eqn (6), (7) and (9), we finally obtain $e=0$, that is, the Jones matrix of the half-wave plate:

$$
\tilde{J}\left(\boldsymbol{b}_{1}\right)=\left(\begin{array}{cc}
0 & f \\
f & 0
\end{array}\right)
$$

The symmetry also allows us to reduce $\tilde{J}(\boldsymbol{k})$, when we calculate the integration in eqn (4) by using eqn (2). We assume that $\theta(\boldsymbol{r})$ and $\delta(\boldsymbol{r})$ have the symmetry of $P 4 m$. The non-vanishing elements are given as

$$
\tilde{J}_{12}\left(\boldsymbol{b}_{1}\right)=-i \frac{1}{\pi^{2}} \int_{0}^{\pi} \mathrm{d} x \int_{0}^{\pi} \mathrm{d} y \exp \left(i \frac{\Gamma}{2}\right) \sin \left(\frac{\Gamma}{2}\right) \sin 2 \theta \sin x \sin y
$$

$$
\begin{aligned}
\tilde{J}_{11}\left(\boldsymbol{b}_{1}-\boldsymbol{b}_{2}\right)= & \frac{1}{2 \pi^{2}} \int_{0}^{\pi} \mathrm{d} x \int_{0}^{\pi} \mathrm{d} y \exp \left(i \frac{\Gamma}{2}\right) \\
& \times\left[\cos \left(\frac{\Gamma}{2}\right)(\cos 2 x+\cos 2 y)\right. \\
& \left.+i \sin \left(\frac{\Gamma}{2}\right) \cos 2 \theta(\cos 2 x-\cos 2 y)\right]
\end{aligned}
$$




$$
\begin{aligned}
\tilde{J}_{22}\left(\boldsymbol{b}_{1}-\boldsymbol{b}_{2}\right)= & \frac{1}{2 \pi^{2}} \int_{0}^{\pi} \mathrm{d} x \int_{0}^{\pi} \mathrm{d} y \exp \left(i \frac{\Gamma}{2}\right) \\
& \times\left[\cos \left(\frac{\Gamma}{2}\right)(\cos 2 x+\cos 2 y)\right. \\
& \left.-i \sin \left(\frac{\Gamma}{2}\right) \cos 2 \theta(\cos 2 x-\cos 2 y)\right] \\
\tilde{J}_{11}(0)=\tilde{J}_{22}(0) & =\frac{1}{\pi^{2}} \int_{0}^{\pi} \mathrm{d} x \int_{0}^{\pi} \mathrm{d} y \exp \left(i \frac{\Gamma}{2}\right) \cos \left(\frac{\Gamma}{2}\right)
\end{aligned}
$$

when $\delta$ is constant and $\tan \theta=\sin y / \sin x$, corresponding to high voltage states, the above equations are reduced;

$$
\begin{gathered}
\tilde{J}_{12}\left(\boldsymbol{b}_{1}\right)=-i \frac{\pi-2}{\pi} \exp \left(i \frac{\Gamma}{2}\right) \sin \left(\frac{\Gamma}{2}\right) \\
\tilde{J}_{11}\left(\boldsymbol{b}_{1}-\boldsymbol{b}_{2}\right)=\tilde{J}_{22}\left(\boldsymbol{b}_{1}-\boldsymbol{b}_{2}\right)=-i \frac{4-\pi}{\pi} \exp \left(i \frac{\Gamma}{2}\right) \sin \left(\frac{\Gamma}{2}\right) \\
\tilde{J}_{11}(0)=\tilde{J}_{22}(0)=\exp \left(i \frac{\Gamma}{2}\right) \cos \left(\frac{\Gamma}{2}\right)
\end{gathered}
$$

Particularly, from eqn (17), it is seen that the diffraction at $\boldsymbol{k}$ $=\boldsymbol{b}_{1}-\boldsymbol{b}_{2}$ plays a role of the half-wave plate as well as that at $\boldsymbol{k}=$ $\boldsymbol{b}_{1}$. In other words, a feature of the spot at $\boldsymbol{b}_{1}-\boldsymbol{b}_{2}$ is that the elliptical polarization can be tuned by the electrical voltage. However, since the grid-like structure simultaneously becomes unstable under a high-voltage, the realization of the tunability is a future work.

\section{Conclusions}

To conclude, we have reported how light is diffracted on a twodimensional micro-structure of nematic liquid crystals. Because the structure is obtained by self-organization, the pattern works as an electrically tunable as well as switchable optical grating. Under a moderate electrical voltage, the intensity of the zerothorder diffraction spot can be tuned, which realizes a high diffraction efficiency. In addition, it is demonstrated that the polarization of the incident light can be converted to different kinds depending on the location of spots. The numerical and theoretical calculations also reproduce well the experiments. Particularly, at some spots, this pattern has a function that inverts the handedness of the circularly polarized light. From the theoretical analysis, it is found that the symmetry of the director field plays important roles for the type of polarization of the diffracted light. These results suggest that further investigations can be performed by changing the birefringence of NLCs and by changing the pattern. Moreover, the combination with the polymer-stabilization technique is interesting. This allows us not only to maintain the director field but also tune the optical properties by external stimuli such as temperature and electric fields, ${ }^{\mathbf{4}, 7}$ which can be applied in the present system..$^{38}$

\section{Conflicts of interest}

There are no conflicts to declare.

\section{Acknowledgements}

This work is supported by the "Hattori-Hokokai" Foundation, the JSPS KAKENHI 18H01851, the NKFIH PD 121019 and FK 125134 grants. P. S., A. B., and F. A. are grateful to the JSPS-HAS bilateral joint research program for the support for their collaboration.

\section{Notes and references}

1 J. Chen, P. J. Bos, H. Vithana and D. L. Johnson, Appl. Phys. Lett., 1995, 67, 2588-2590.

2 D. Subacius, P. J. Bos and O. D. Lavrentovich, Appl. Phys. Lett., 1997, 71, 1350-1352.

3 N. Kawatsuki, T. Hasegawa, H. Ono and T. Tamoto, Adv. Mater., 2003, 15, 991-994.

4 H. Ren, Y.-H. Fan and S.-T. Wu, Appl. Phys. Lett., 2003, 82, 3168-3170.

5 H. Ono, A. Emoto, F. Takahashi, N. Kawatsuki and T. Hasegawa, J. Appl. Phys., 2003, 94, 1298-1303.

6 B. I. Senyuk, I. I. Smalyukh and O. D. Lavrentovich, Opt. Lett., 2005, 30, 349.

7 J. Yan, Y. Li and S.-T. Wu, Opt. Lett., 2011, 36, 1404.

8 H. Chen, G. Tan, Y. Huang, Y. Weng, T.-H. Choi, T.-H. Yoon and S.-T. Wu, Sci. Rep., 2017, 7, 39923.

9 J.-H. Park, C.-J. Yu, J. Kim, S.-Y. Chung and S.-D. Lee, Appl. Phys. Lett., 2003, 83, 1918-1920.

10 M. Le Doucen and P. Pellat-Finet, Opt. Commun., 1998, 151, 321-330.

11 D. Xu, G. Tan and S.-T. Wu, Opt. Express, 2015, 23, 12274.

12 A. Ryabchun, A. Bobrovsky, Y. Gritsai, O. Sakhno, V. Shibaev and J. Stumpe, ACS Appl. Mater. Interfaces, 2015, 7, 25542560.

13 Z. He, T. Nose and S. Sato, Jpn. J. Appl. Phys., Part 1, 1996, 35, 3529-3530.

14 J.-L. Zhu, J.-G. Lu, J. Qiang, E.-W. Zhong, Z.-C. Ye, Z. He, X. Guo, C.-Y. Dong, Y. Su and H.-P. D. Shieh, J. Appl. Phys., 2012, 111, 033101.

15 B. Wen, R. G. Petschek and C. Rosenblatt, Appl. Opt., 2002, 41, 1246.

16 W. M. Gibbons and S. Sun, Appl. Phys. Lett., 1994, 65, 25422544.

17 H. Yoshida, K. Asakura, J. Fukuda and M. Ozaki, Nat. Commun., 2015, 6, 7180.

18 Y. Guo, M. Jiang, C. Peng, K. Sun, O. Yaroshchuk, O. Lavrentovich and Q. H. Wei, Adv. Mater., 2016, 28, 2353-2358.

19 H.-C. Jau, T.-H. Lin, Y.-Y. Chen, C.-W. Chen, J.-H. Liu and A. Y.-G. Fuh, Appl. Phys. Lett., 2012, 100, 131909.

20 Y. Xiang, H.-Z. Jing, Z.-D. Zhang, W.-J. Ye, M.-Y. Xu, E. Wang, P. Salamon, N. Éber and Á. Buka, Phys. Rev. Appl., 2017, 7, 064032.

21 N. Vaupotič, M. Ali, P. W. Majewski, E. Gorecka and D. Pociecha, ChemPhysChem, 2018, 19, 2566-2571. 
22 R. B. Meyer, Phys. Rev. Lett., 1969, 22, 918-921.

23 P. Pieranski, E. Dubois-Violette and E. Guyon, Phys. Rev. Lett., 1973, 30, 736-739.

24 M. R. Kuzma, Phys. Rev. Lett., 1986, 57, 349-352.

25 A. Buka and L. Kramer, Pattern formation in liquid crystals, Springer New York, New York, NY, 1996.

26 L. K. Migara and J.-K. Song, NPG Asia Mater., 2018, 10, e459.

27 H.-C. Yeh, G.-H. Chen, C.-R. Lee and T.-S. Mo, J. Chem. Phys., 2007, 127, 141105.

28 S.-W. Kang and L.-C. Chien, Appl. Phys. Lett., 2007, 90, 221110.

29 A. Ryabchun and A. Bobrovsky, Adv. Opt. Mater., 2018, 6, 1800335.

30 Y. Sasaki, V. Jampani, C. Tanaka, N. Sakurai, S. Sakane, K. V. Le, F. Araoka and H. Orihara, Nat. Commun., 2016, 7, 13238.
31 A. Rapini, J. Phys., 1973, 34, 629-633.

32 A. Rapini, L. Léger and A. Martinet, J. Phys. Colloq., 1975, 36, C1-189-C1-196.

33 E. Brasselet and C. Loussert, Opt. Lett., 2011, 36, 719.

34 E. Brasselet, Phys. Rev. Lett., 2012, 108, 087801.

35 P. Salamon, N. Éber, Y. Sasaki, H. Orihara, Á. Buka and F. Araoka, Phys. Rev. Appl., 2018, 10, 044008.

36 P. Oswald, G. Poy and A. Dequidt, Liq. Cryst., 2017, 44, 969988.

37 S. Dhara, J. K. Kim, S. M. Jeong, R. Kogo, F. Araoka, K. Ishikawa and H. Takezoe, Phys. Rev. E: Stat., Nonlinear, Soft Matter Phys., 2009, 79, 060701(R).

38 Y. Sasaki, M. Ueda, K. V. Le, R. Amano, S. Sakane, S. Fujii, F. Araoka and H. Orihara, Adv. Mater., 2017, 29, 1703054. 